\title{
Percepción y experiencias sobre el ciberbullying en estudiantes universitarios
}

\author{
Perception and Experiences About Cyberbulling in University Students
}

\author{
Marisol Rodríguez Correa \\ Universidad Internacional de La Rioja \\ marisol.rodriguez@unir.net
}

Juan Carlos Rivadulla López

Universidad de la Coruña

juan.rivadulla@udc.es

Fecha presentación: 01/08/2018 | Aceptación: 19/11/2018 |Publicación: 0/0/2018

\section{Resumen}

En la actualidad nos encontramos con un alto porcentaje de uso de las TIC por parte de los adolescentes, principalmente en lo que se refiere a la comunicación on-line. Las amenazas, burlas, suplantación de la identidad y el envío de material con contenido sexual, son algunas de las múltiples formas a través de las cuales se manifiesta el cyberbullying, siendo Internet y el teléfono móvil los principales medios utilizados por los jóvenes para hacerlo. El objetivo de este estudio fue conocer la percepción y experiencia de un grupo de jóvenes universitarios sobre el ciberbullying. Para ello contamos con la participación de 864 estudiantes de seis universidades del noroeste de España. Se ha seguido una metodología cuantitativa de carácter no experimental de tipo encuesta; concretamente se utilizó un muestreo no probabilístico, accidental o incidental, condicionado por la disponibilidad de los sujetos a participar en el estudio. La mayoría del alumnado indicó que el acoso a través del móvil y de Internet tiene más efecto sobre la víctima que el "bullying tradicional", indicando que nunca han sufrido acoso ni han ejercido como acosadores a través de Internet y del móvil; y solo un menor porcentaje de estudiantes expresó que sí ha sufrido acoso a través del móvil, pero con poca frecuencia. A la mayoría de los hombres que indicaron que sufren acoso a través de Internet les preocupa lo que los demás puedan pensar sobre lo que les está sucediendo, en el caso de las mujeres consultadas, la mayoría indicaron que cuando les acosan se sienten solas y les preocupa igualmente lo que los demás puedan pensar sobre lo que les está sucediendo. Debemos educar y sensibilizar a la sociedad en general acerca del tema del acoso y ciberacoso, implementando medidas educativas para su prevención en los centros educativos desde los primeros niveles.

Palabras clave: TIC, cyberbullying, universidad, estudiantes.

\section{Resum}

En l'actualitat ens trobem amb un alt percentatge d'ús de les TIC per part dels adolescents, principalment pel que fa a la comunicació on-line. Les amenaces, burles, suplantació de la identitat i l'enviament de material amb contingut sexual, són algunes de les múltiples formes a través de les quals es manifesta el cyberbullying, sent l'Internet i el telèfon mòbil els principals mitjans utilitzats pels joves per fer-ho. L'objectiu d'aquest estudi va ser conèixer la percepció i experiència d'un grup de joves universitaris sobre el ciberbullying, comptant amb la participació de 864 estudiants de sis Universitats del nord-oest d'Espanya. S'ha seguit una metodologia quantitativa de caràcter no experimental de tipus enquesta, concretament es va utilitzar un mostreig no probabilístic, accidental o incidental, condicionat per la disponibilitat dels subjectes a participar en l'estudi. La majoria de l'alumnat consultat va indicar que l'assetjament a través del mòbil i d'Internet té més efecte sobre la víctima que el "bullying tradicional", indicant que mai han patit assetjament ni han exercit com assetjadors a través d'Internet i del mòbil; i tret d'un menor percentatge d'estudiants va expressar que sí que ha patit assetjament a través del mòbil, però amb poca freqüència. La majoria dels homes consultats que van indicar que pateixen assetjament a través d'Internet els preocupa el que els altres puguin pensar sobre el que els està succeint, en el cas de les dones consultades, la majoria van indicar que quan els assetgen se senten soles i els preocupa igualment el que els altres puguin pensar sobre el que els està succeint. Hem d'educar i sensibilitzar la societat en general sobre el tema de l'assetjament i ciberassetjament, implementant mesures educatives per a la seva prevenció en els centres educatius des dels primers nivells. 
Rodríguez Correa, Marisol; Rivadulla López, Juan Carlos. (2018). "Percepción y experiencias sobre el ciberbullying en estudiantes universitarios” en @tic. revista d'innovació educativa. Número 21 Otoño (Julio-Diciembre 2018), pp. 10-22.

\begin{abstract}
Nowadays there is a high percentage of ICT use by adolescents, mostly in terms of online communication. Threats, taunts, spoofing the identity and the sending material with sexual content are some of the ways through which cyberbullying manifests, the Internet and mobile phone to be the main media used by young people to do so. The objective of this study was to know the perception and experience of a group of university students about cyberbullying. With that goal we count with the participation of 864 students from six universities of northwestern Spain. A nonexperimental quantitative methodology of a survey type was followed, specifically a non-probabilistic, accidental or incidental sampling was used, conditioned by the availability of subjects to participate in the study. The majority of the students indicated that harassment through mobile phones and the Internet has more effect on the victim than "traditional bullying", indicating that they have never suffered harassment or have exercised as stalkers through the Internet and mobile phones; and only a lower percentage of students expressed that they have suffered harassment through mobile phones, but infrequently. The majority of the men consulted who indicated that they suffer harassment through the Internet worry about what others may think about what is happening to them, in the case of the women consulted, most indicated that when they are harassed they feel alone and they It also worries what others may think about what is happening to them. We must educate and sensitize society in general about the subject of harassment and cyberbullying, implementing educational measures for its prevention in schools since the first levels.
\end{abstract}

Key Words: TIC, cyberbullying, university, students.

\section{Introducción}

El acoso es definido como una forma específica de violencia escolar, donde uno o varios agresores con intencionalidad causan dolor, acosan y someten de forma reiterada a otro compañero (Avilés, 2002; Ortega y Mora-Merchán, 2008; Cerezo, 2009; Álvarez-García, Núñez, Rodríguez, Álvarez y Dobarro, 2011).

En la actualidad los medios tecnológicos y las redes digitales se encuentran relacionados íntimamente con nuestros jóvenes, lo que ha generado una cultura del ciberespacio y nuevas formas de comunicación, siendo Internet y la telefonía móvil las más usadas. El ciberacoso es definido por Belsey (2005) como el uso de algunas Tecnologías de la Información y Comunicación para manifestar comportamientos vejatorios y difamatorios de un individuo o un grupo, que pretende dañar a otro de forma repetitiva y hostil. Por lo tanto, ya no se requiere un espacio físico directo del agresor con la víctima para que ocurra intimidación, el uso intensivo de la red al propiciar estas relaciones sociales, incluye igualmente la posibilidad de perjudicar a otros o ser perjudicado (Sabater y López-Hernáez, 2015).

Actualmente Internet y las tecnologías de la información y de la comunicación (TIC) están teniendo una gran presencia en la vida de las personas, cerca del $52 \%$ de la población europea hace uso de los espacios on-line según un estudio realizado por Eurostat (2017), y con relación a la telefonía móvil en Europa existen 106 líneas telefónicas por cada 100 habitantes (Eurostat, 2017).

El rápido crecimiento de las $\mathrm{TIC}$ ha traído consigo numerosos beneficios, pero sin duda también perjuicios (Cabello, 2013), siendo uno de ellos el ciberacoso, el cual está tomando cada día más fuerza en nuestras sociedades. Según los resultados de un estudio realizado por Oliveros, Amemiya, Condorimay, Oliveros, Barrientos y Rivas (2012), tener teléfono móvil, ordenador en el cuarto, acceso a Internet fuera de casa y una mayor solvencia económica fueron algunos de los factores de riesgo de ciberbullying, tanto en el rol de víctima como en el de acosador.

De la misma forma, los datos arrojados por el estudio sobre hábitos seguros en el uso de Smartphones por los niños y adolescentes españoles del Observatorio de la Seguridad de la Información de INTECO (2011), señalan que el $2,5 \%$ de los menores consultados confiesa haber sido objeto de acoso por parte de otros menores, a través del Smartphone, y el 0,8\% de jóvenes reconoce haberlo realizado de manera activa, en forma de insultos o amenazas a través de su teléfono móvil.

Algunas de las múltiples formas a través de las cuales se manifiesta el ciberacoso son las amenazas, burlas, suplantación de la identidad y el envío de material con contenido sexual, siendo Internet y el teléfono móvil los principales medios utilizados por los jóvenes para hacerlo (Calvete, Orue, Estévez, Villardón, \& Padilla, 2010; Garaigordobil \& Aliri, 2013; Durán-Segura \& Martínez-Pecino, 2015).

Los estudios sobre el acoso y ciberacoso se encuentran en un estado inicial, las primeras investigaciones llevadas a cabo sobre la violencia entre iguales a través de las TIC se llevaron a cabo en países como EEUU, Australia, Reino Unido, Bélgica e Italia (Finkelhor, Mitchell, y Wolak, 2007; Vandebosch y Van Cleemput, 2009; Tangen \& Campbell, 2010; Palladino, Nocentini, y Menesini, 2012). En España, igualmente podemos mencionar investigaciones sobre esta temática como las realizadas por Defensor del Pueblo-Unicef, 2007; Avilés, 2009; Bringué y Sádaba, 2010; Calmaestra, 2011; Garaigordobil, 2012; Del Rey, Mora-Merchán, Casas, Ortega-Ruiz y Elipe, 2018; Giménez, Arnaiz, CerezoRamírez y Prodócimo, 2018; Yubero, Larrañaga, Navarro y Elche, 2018, entre otras.

Algunos estudios han demostrado que los jóvenes que se enfrentan al ciberacoso manifiestan mayores niveles de problemas psicosomáticos que aquellos no implicados en situaciones similares (Beckman, Hagquist, \& Hellstrom, 2012). De la misma forma, otras investigaciones sobre el tema indican que los jóvenes que sufren ciberacoso presentan mayores niveles de síntomas depresivos (Nixon, 2014), menor autoestima (0`Brien \& Moules, 2013), y mayores niveles de ansiedad (Sontag, Clemans, Graber, \& Lyndon, 2011). Otros estudios como el de Muñoz (2016), indican que, en los centros escolares españoles, hay menores que presentan dificultad en la integración escolar y en el proceso de aprendizaje porque afirman tener miedo y sentirse indefensos ante la amenaza, la violencia y el dominio de su agresor. El ciberacoso por lo tanto, se ha convertido en un fenómeno tan problemático socialmente en España según éste y otros estudios, que se ha establecido una reglamentación para proteger al menor de los delitos de tipo cibernético, desarrollada en 
Rodríguez Correa, Marisol; Rivadulla López, Juan Carlos. (2018). "Percepción y experiencias sobre el ciberbullying en estudiantes universitarios” en @tic. revista d'innovació educativa. Número 21 Otoño (Julio-Diciembre 2018), pp. 10-22.

el artículo 197 del Código Penal y modificado en las Leyes Orgánicas 5/2010 de 22 de junio, 3/2011 de 28 de enero y $1 / 2015$ de 30 de marzo (Fundación MutuaMadrileña y la Fundación Anar, 2016).

Con relación a la educación superior, estudios como el de López (2017) sobre el ciberbullying en la universidad, indican que existe relación entre licenciatura y acoso escolar, así como entre sexo y victimización verbal. Por otra parte, los estudiantes de las licenciaturas de Ciencias Sociales encuestados presentaron más acoso escolar, así como victimización relacional y victimización verbal en comparación con los de Ciencias de la Salud. Finalmente, los resultados de este estudio confirman que el acoso escolar y el acoso cibernético son fenómenos que se presentan dentro de las instituciones de nivel superior.

Si bien es cierto que existen muchos estudios acerca del Bullying o ciberbullying en estudiantes de Secundaria (Cerezo, 2009; Palacios, Polo, Felipe, León, y Fajardo, 2013; Durán-Segura y Martínez-Pecino, 2015; Garaigordobil, 2015; Garaigordobil, Martínez, y Machimbarrena, 2017; Del Rey et al. 2018; Giménez et al., 2018; Yubero, et al., 2018), cuando nos referimos a la experiencia del bullying o ciberbullying en estudiantes universitarios encontramos escasas investigaciones sobre el tema.

Por este motivo pretendemos realizar un estudio para conocer la percepción y la experiencia de un grupo de jóvenes universitarios con relación al ciberbullying, pues se trata de uno de los sectores poblacionales que podría verse involucrado en esta problemática. Sin duda alguna, a partir del uso frecuente de Internet o móviles de última generación por parte de estos jóvenes, conoceremos la concepción y percepción que ellos puedan tener sobre el ciberbullying y la cantidad de veces que han sufrido o han ejercido acoso, siendo ello relevante en términos de experiencia cotidiana y de proximidad con los fenómenos estudiados.

\section{Método}

El interés principal en este estudio fue conocer la percepción y experiencia de un grupo de jóvenes universitarios sobre el ciberbullying, entendido como una forma específica de violencia escolar, donde uno o varios agresores con mayor poder y con intencionalidad causan dolor, acosan y someten reiteradamente a un compañero/a.

Teniendo en cuenta el contenido que aborda este estudio y por motivos de protección de datos, se prefiere mantener en el anonimato el nombre de las universidades donde se llevó a cabo la investigación, indicando que la recogida de datos se realizó a principios del curso académico 2016/2017 de forma presencial y en formato papel en seis universidades del noroeste de España. Para ello, se contactó con los responsables de las instituciones educativas para obtener el permiso necesario $y$, posteriormente con los docentes que tendrían que permitir acudir a sus clases para que su alumnado cubriera el cuestionario. Cabe destacar que, en algunos casos, fueron los propios docentes (ajenos a la investigación) los que distribuyeron el cuestionario, lo cual nos facilitó el trabajo a los investigadores. Los cuestionarios se devolvieron de la misma forma que lo recibieron (en formato papel y de forma presencial). La participación fue voluntaria y se garantizó el anonimato y la confidencialidad de los datos obtenidos. Para responderlo, se les concedió el tiempo que ellos consideraron oportuno, teniendo una duración media de
30 minutos.

\subsection{Participantes}

Para la realización de esta investigación hemos contado con la participación de 864 estudiantes, de los cuales la mayoría son mujeres (70,83\%) de entre 18 y 20 años (55,09\%), estudian algún grado relacionado con las Ciencias Sociales y Jurídicas (36,23\%) y la mayoría viven en un entorno urbano (60,76\%). Además, todos los participantes disponen de teléfono móvil, así como conexión a Internet desde casa y desde el móvil (ver tabla 1).

\begin{tabular}{|c|c|c|c|}
\hline & & $F$ & $\%$ \\
\hline \multirow{6}{*}{ 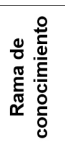 } & Artes y humanidades & 106 & 12,27 \\
\hline & Ciencias & 227 & 26,27 \\
\hline & Ciencias de la salud & 129 & 14,93 \\
\hline & Ciencias sociales y jurídicas & 313 & 36,23 \\
\hline & Ingenieria y Arquitectura & 89 & 10,30 \\
\hline & TOTAL & 864 & 100 \\
\hline \multirow{3}{*}{ 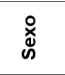 } & Hombres & 252 & 29,17 \\
\hline & Mujeres & 612 & 70,83 \\
\hline & TOTAL & 864 & 100 \\
\hline \multirow{5}{*}{$\begin{array}{l}\text { Dृ } \\
\text { 吾 }\end{array}$} & De 18 a 20 años & 476 & 55,09 \\
\hline & De 21 a 23 años & 221 & 25,58 \\
\hline & De 24 a 26 años & 99 & 11,46 \\
\hline & Mayores de 27 años & 68 & 7,87 \\
\hline & TOTAL & 864 & 100 \\
\hline \multirow{3}{*}{ 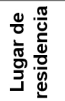 } & Urbano & 525 & 60,76 \\
\hline & Rural & 339 & 39,24 \\
\hline & TOTAL & 864 & 100 \\
\hline \multirow{6}{*}{ 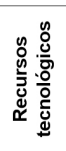 } & Dispone de teléfono móvil & 864 & 100 \\
\hline & TOTAL & 864 & 100 \\
\hline & Dispone de Internet en casa & 864 & 100 \\
\hline & TOTAL & 864 & 100 \\
\hline & Dispone de Internet en el móvil & 864 & 100 \\
\hline & TOTAL & 864 & 100 \\
\hline
\end{tabular}

Tabla 1. Distribución de los participantes.

\subsection{Instrumento}

Se realizó una adaptación del cuestionario propuesto por Ortega, Calmaestra, y Mora-Merchán (2007), el cual mide las relaciones entre los jóvenes a través de las nuevas tecnologías (Teléfono Móvil e Internet), así como también el acoso que dicen sufrir o ejercer dichos jóvenes a través de estas tecnologías.

Los apartados que incluyó el cuestionario se elaboraron en torno al objetivo que nos marcamos para esta investigación y, por supuesto, teniendo en cuenta toda la fundamentación teórica expuesta anteriormente. Este cuestionario fue revisado por tres expertos en la materia, con el fin de validar su contenido y su inteligibilidad para el participante, realizando las modificaciones oportunas. Teniendo en cuenta las correcciones realizadas, se aplicó el instrumento a una muestra piloto de 25 estudiantes ( 5 de cada rama de conocimiento), con la finalidad de detectar problemas de funcionamiento de los ítems antes de su aplicación a la totalidad de la muestra (Expósito, Navarro, Thoilliez, y López, 2010), pero el resultado fue óptimo y no se necesitó realizar más cambios.

En sus aspectos formales, el cuestionario se compone de 8 preguntas de identificación (edad, sexo, rama de conocimiento...) y 19 preguntas de opción múltiple que recaban información sobre contenidos relacionados con las situaciones de acoso entre universitarios a través del teléfono móvil e Internet (ver anexo). Las preguntas que se han incluido han tratado de identificar a las víctimas y a los agresores a través de móvil e Internet, así como determinar la frecuencia de participación en este papel. Se pretende averiguar: la percepción que tienen los participantes sobre los efectos del ciberbullying (3 preguntas); la frecuencia del acoso sufrido o ejercido a través del teléfono móvil e internet (cuatro preguntas - 
Rodríguez Correa, Marisol; Rivadulla López, Juan Carlos. (2018). "Percepción y experiencias sobre el ciberbullying en estudiantes universitarios” en @tic. revista d'innovació educativa. Número 21 Otoño (Julio-Diciembre 2018), pp. 10-22.

dos para móvil y dos para Internet) y la percepción que tienen ante el acoso (12 preguntas -seis para móvil y seis para Internet).

\subsection{Procedimiento de análisis}

Para dar respuesta a la intencionalidad exploratoria y descriptiva de este estudio (Bisquerra, 2004), se ha utilizado una metodología cuantitativa de carácter no experimental de tipo encuesta (McMillan y Schumacher, 2005). Concretamente se utilizó un muestreo no probabilístico, accidental o incidental, condicionado por la disponibilidad de los sujetos a participar en el estudio. Este tipo de muestreos, aunque no permiten "extraer muestras representativas de la población, sí facilitan el estudio cualitativo en profundidad del tema que interesa dentro de un contexto determinado" (Martínez, 2007, p. 56). Como condiciones para poder colaborar en este estudio nos propusimos que los participantes utilizaran habitualmente Internet (tanto desde el PC como desde el móvil) y que llevaran cursando como mínimo un año en la Universidad. Este último dato nos aseguraba que los participantes tuvieran tiempo suficiente para haber padecido o ejercido el acoso en el ámbito universitario. El análisis de las respuestas recogidas en el cuestionario se analizó de forma directa, estableciéndose porcentajes, y fue realizado independientemente por dos investigadores, discutiendo las posibles discrepancias. Además, se aplicó estadística descriptiva y la prueba estadística de independencia de criterios Chi-cuadrado con $95 \%$ de confianza para determinar la asociación entre las variables de interés (sexo, edad, rama de conocimiento y lugar de residencia), y las categorías de acoso sufrido y ejercido a través de Internet y del teléfono móvil. Este análisis estadístico se realizó con el programa SPSS 24.

Para elaborar y sintetizar los resultados, lo primero que se hizo fue identificar el número de personas que indicaron haber sufrido acoso a través del móvil o de Internet (apartado a). A continuación, y tomando como referente los tres grandes apartados que se incluyen en el cuestionario, se procedió a analizar las respuestas a las preguntas: b) percepción sobre los efectos del ciberbullying; c) frecuencia del acoso sufrido o ejercido a través del teléfono móvil e internet; y d) percepción ante el acoso.

\section{Resultados}

\section{a) Identificación de los participantes en cuanto al acoso sufrido y ejercido}

En general, los datos muestran que pocos son los participantes que confirman haber sufrido acoso a través de Internet $y$, aún menos, los que indican haberlo ejercido (tabla 2). No obstante, entre los que indican que han sufrido o ejercido acoso se puede establecer un tipo perfil atendiendo a la mayoría porcentual, siendo este en ambos casos el de una mujer de entre 18 y 20 años que estudia alguna carrera de la rama de conocimiento de ciencias sociales y jurídicas y que vive en un entorno urbano.

Los resultados muestran que existen diferencias significativas entre el acoso sufrido y el ejercido a través de Internet por los participantes y el sexo $(p=0,019)$, la edad $(p=0,009)$, la rama del conocimiento $(p=0,041)$ y el lugar de residencia $(p=0,013)$.

\begin{tabular}{|c|c|c|c|c|c|c|c|}
\hline & & \multicolumn{3}{|c|}{ Acoso sufrido } & \multicolumn{3}{|c|}{ Acoso ejercido } \\
\hline & & Si & No & $x^{2}$ & Si & No & $x^{2}$ \\
\hline \multirow{2}{*}{ 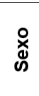 } & Hombres & $\begin{array}{c}72 \\
(8.33)\end{array}$ & $\begin{array}{c}180 \\
(20,83)\end{array}$ & \multirow[b]{2}{*}{,023 } & $\begin{array}{c}10 \\
(1,16)\end{array}$ & $\begin{array}{c}242 \\
(28,01)\end{array}$ & \multirow{2}{*}{,019 } \\
\hline & Mujeres & $\begin{array}{c}108 \\
(12,50)\end{array}$ & $\begin{array}{c}504 \\
(58,33)\end{array}$ & & $\begin{array}{c}72 \\
(8.33)\end{array}$ & $\begin{array}{c}540 \\
(62,50)\end{array}$ & \\
\hline \multirow{4}{*}{ 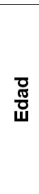 } & De 18 a 20 años & $\begin{array}{c}138 \\
(15,97)\end{array}$ & $\begin{array}{c}338 \\
(39,12)\end{array}$ & \multirow{4}{*}{017} & $\begin{array}{c}45 \\
(5,21) \\
\end{array}$ & $\begin{array}{c}431 \\
(49,88)\end{array}$ & \multirow{4}{*}{,009 } \\
\hline & De 21 a 23 años & $\begin{array}{c}35 \\
(4,05)\end{array}$ & $\begin{array}{c}186 \\
(21,53)\end{array}$ & & $\begin{array}{c}31 \\
(3,59)\end{array}$ & $\begin{array}{c}190 \\
(21,99)\end{array}$ & \\
\hline & De 24 a 26 años & $\begin{array}{c}5 \\
(0,58)\end{array}$ & $\begin{array}{c}94 \\
(10,88)\end{array}$ & & $\begin{array}{c}5 \\
(0,58)\end{array}$ & $\begin{array}{c}94 \\
(10,88)\end{array}$ & \\
\hline & Mayores de 27 años & $\begin{array}{c}2 \\
2 \\
(0,23)\end{array}$ & $\begin{array}{c}66 \\
(7,64)\end{array}$ & & $\begin{array}{c}1 \\
(0,12)\end{array}$ & $\begin{array}{c}67 \\
(7,75)\end{array}$ & \\
\hline \multirow{5}{*}{ 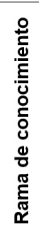 } & Artes y humanidades & $\begin{array}{c}11 \\
(1,27)\end{array}$ & $\begin{array}{c}95 \\
(11,00)\end{array}$ & \multirow{5}{*}{,012 } & $\begin{array}{c}11 \\
(1,27)\end{array}$ & $\begin{array}{c}95 \\
(11,00)\end{array}$ & \multirow{5}{*}{,041 } \\
\hline & Ciencias & $\begin{array}{c}42 \\
(4,86)\end{array}$ & $\begin{array}{c}185 \\
(21,41)\end{array}$ & & $\begin{array}{c}25 \\
(2,89)\end{array}$ & $\begin{array}{c}202 \\
(23,38)\end{array}$ & \\
\hline & Ciencias de la salud & $\begin{array}{c}13 \\
(1,50)\end{array}$ & $\begin{array}{c}116 \\
(13,43)\end{array}$ & & $\begin{array}{c}9 \\
(1,04)\end{array}$ & $\begin{array}{c}120 \\
(13,89)\end{array}$ & \\
\hline & Ciencias sociales y jurídicas & $\begin{array}{c}111 \\
(12,85)\end{array}$ & $\begin{array}{c}202 \\
(23,38)\end{array}$ & & $\begin{array}{c}27 \\
(3,13)\end{array}$ & $\begin{array}{c}286 \\
(33,10)\end{array}$ & \\
\hline & Ingeniería y Arquitectura & $\begin{array}{c}3 \\
(0,35)\end{array}$ & $\begin{array}{c}86 \\
(9,95)\end{array}$ & & $\begin{array}{c}10 \\
(1,16)\end{array}$ & $\begin{array}{c}79 \\
(9,14)\end{array}$ & \\
\hline \multirow{2}{*}{ 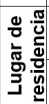 } & Urbano & $\begin{array}{c}160 \\
(18,52)\end{array}$ & $\begin{array}{c}365 \\
(42,25)\end{array}$ & \multirow{2}{*}{011} & $\begin{array}{c}53 \\
(6,13)\end{array}$ & $\begin{array}{c}472 \\
(54,63)\end{array}$ & \multirow{2}{*}{,013 } \\
\hline & Rural & $\begin{array}{c}20 \\
(2,31)\end{array}$ & $\begin{array}{c}319 \\
(36,92)\end{array}$ & & $\begin{array}{c}19 \\
(2,20)\end{array}$ & $\begin{array}{c}320 \\
(37,04)\end{array}$ & \\
\hline
\end{tabular}

Tabla 2. Frecuencia/porcentaje de participantes $(n=864)$ y Chi Cuadrado de Pearson $\left(X^{2}, p<0,05\right)$ en relación al acoso sufrido y al ejercido a través de Internet

Por otro lado, el número de participantes que confirman haber sufrido o ejercido acoso a través del teléfono móvil es más elevado si lo comparamos con el de Internet (tabla 3). De la misma forma, entre los participantes que indican que han sufrido o ejercido acoso a través del teléfono móvil, se puede establecer un tipo perfil atendiendo a la mayoría porcentual, los datos son idénticos a los obtenidos en el caso de Internet, pues este perfil sigue siendo en ambos casos (acoso sufrido y ejercido) el de una mujer de entre 18 y 20 años que estudia alguna carrera de la rama de conocimiento de ciencias sociales y jurídicas y que vive en un entorno urbano.

Los resultados muestran que existen diferencias significativas entre el acoso sufrido y el ejercido a través del móvil por los participantes y el sexo $(p=0,010)$, la edad $(p=0,031)$, la rama del conocimiento $(p=0,018)$ y el lugar de residencia $(p=0,028)$.

\begin{tabular}{|c|c|c|c|c|c|c|c|}
\hline & & \multicolumn{3}{|c|}{ Acoso sufrido } & \multicolumn{3}{|c|}{ Acoso ejercido } \\
\hline & & Sí & No & $x^{2}$ & Si & No & $x^{2}$ \\
\hline \multirow{2}{*}{ 离 } & Hombres & $\begin{array}{c}74 \\
(8,56)\end{array}$ & $\begin{array}{c}178 \\
(20,60)\end{array}$ & \multirow[b]{2}{*}{002} & $\begin{array}{c}39 \\
(4.51)\end{array}$ & $\begin{array}{c}213 \\
(24,65)\end{array}$ & \multirow[b]{2}{*}{,010 } \\
\hline & Mujeres & $\begin{array}{c}187 \\
(21,64)\end{array}$ & \begin{tabular}{|c|}
425 \\
$(49,19)$
\end{tabular} & & $\begin{array}{c}115 \\
(13,31)\end{array}$ & \begin{tabular}{|c|}
497 \\
$(57.52)$
\end{tabular} & \\
\hline \multirow{4}{*}{ 嵒 } & De 18 a 20 años & $\begin{array}{c}159 \\
(18,40)\end{array}$ & $\begin{array}{c}317 \\
(36,69)\end{array}$ & \multirow{4}{*}{007} & $\begin{array}{c}94 \\
(10,88)\end{array}$ & $\begin{array}{c}382 \\
(44,21)\end{array}$ & \multirow{4}{*}{ 031 } \\
\hline & De 21 a 23 años & $\begin{array}{c}83 \\
(9,61)\end{array}$ & $\begin{array}{c}138 \\
(15,97)\end{array}$ & & $\begin{array}{c}39 \\
(4,51)\end{array}$ & $\begin{array}{c}182 \\
(21,06)\end{array}$ & \\
\hline & De 24 a 26 años & $\begin{array}{c}16 \\
(1,85)\end{array}$ & $\begin{array}{c}83 \\
(9,61)\end{array}$ & & $\begin{array}{c}19 \\
(2,20)\end{array}$ & $\begin{array}{c}80 \\
(9,26)\end{array}$ & \\
\hline & Mayores de 27 años & $\begin{array}{c}3 \\
(0,35)\end{array}$ & $\begin{array}{c}65 \\
(7,52)\end{array}$ & & $\begin{array}{c}2 \\
(0,23)\end{array}$ & $\begin{array}{c}66 \\
(7,64)\end{array}$ & \\
\hline \multirow{5}{*}{ 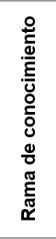 } & Artes y humanidades & $\begin{array}{c}29 \\
(3,36)\end{array}$ & $\begin{array}{c}77 \\
(8,91)\end{array}$ & \multirow{5}{*}{011} & $\begin{array}{c}21 \\
(2,43)\end{array}$ & $\begin{array}{c}85 \\
(9,84)\end{array}$ & \multirow{5}{*}{,018 } \\
\hline & Ciencias & $\begin{array}{c}62 \\
(7,18)\end{array}$ & $\begin{array}{c}165 \\
(19,10)\end{array}$ & & $\begin{array}{c}50 \\
(5,79)\end{array}$ & $\begin{array}{c}177 \\
(20,49)\end{array}$ & \\
\hline & Ciencias de la salud & $\begin{array}{c}38 \\
(4,40)\end{array}$ & $\begin{array}{c}91 \\
(10,53)\end{array}$ & & $\begin{array}{c}18 \\
(2,08)\end{array}$ & $\begin{array}{c}111 \\
(12,85)\end{array}$ & \\
\hline & Ciencias sociales y jurídicas & $\begin{array}{c}124 \\
(14,35)\end{array}$ & $\begin{array}{c}189 \\
(21,88)\end{array}$ & & $\begin{array}{c}59 \\
(6,83)\end{array}$ & $\begin{array}{c}254 \\
(29,40)\end{array}$ & \\
\hline & Ingeniería y Arquitectura & $\begin{array}{c}7 \\
(0,81)\end{array}$ & $\begin{array}{c}82 \\
(9,49)\end{array}$ & & $\begin{array}{c}6 \\
(0,69)\end{array}$ & $\begin{array}{c}83 \\
(9,61)\end{array}$ & \\
\hline \multirow{2}{*}{ 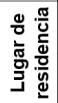 } & Urbano & $\begin{array}{c}193 \\
(22,34)\end{array}$ & $\begin{array}{c}332 \\
(38,43)\end{array}$ & \multirow{2}{*}{003} & $\begin{array}{c}92 \\
(10,65)\end{array}$ & $\begin{array}{c}433 \\
(50,12)\end{array}$ & \multirow{2}{*}{028} \\
\hline & Rural & $\begin{array}{c}68 \\
(7,87)\end{array}$ & $\begin{array}{c}271 \\
(31,37)\end{array}$ & & $\begin{array}{c}62 \\
(7,18)\end{array}$ & $\begin{array}{c}277 \\
(32,06)\end{array}$ & \\
\hline
\end{tabular}

Tabla 3. Frecuencia/porcentaje de participantes $(n=864)$ y Chi Cuadrado de Pearson $\left(X^{2}, p<0,05\right)$ en relación al acoso sufrido y al ejercido a través del teléfono móvil 
Rodríguez Correa, Marisol; Rivadulla López, Juan Carlos. (2018). "Percepción y experiencias sobre el ciberbullying en estudiantes universitarios” en @tic. revista d'innovació educativa. Número 21 Otoño (Julio-Diciembre 2018), pp. 10-22.

\section{b) Percepción sobre los efectos del ciberbullying}

En cuanto a la percepción sobre el acoso que tienen los estudiantes universitarios (figura 1), la mayoría considera que el acoso a través del móvil $(46,57 \%$ de mujeres y $42,46 \%$ de hombres) y de Internet $(58,82 \%$ de mujeres y $42,86 \%$ de hombres) tiene más efecto sobre la víctima que el "bullying tradicional". Además, la mayoría consideran que prohibir los móviles en las instituciones educativas no ayudaría a evitar el acoso porque utilizarían estos dispositivos en secreto $(52,94 \%$ de mujeres y $64,29 \%$ de hombres) o fuera de la Universidad $(41,18 \%$ de mujeres y $28,57 \%$ de hombres). Cabe destacar que un porcentaje mínimo de estudiantes (menos del 8\%) considera que sí serviría el prohibir el uso de móviles en los centros.

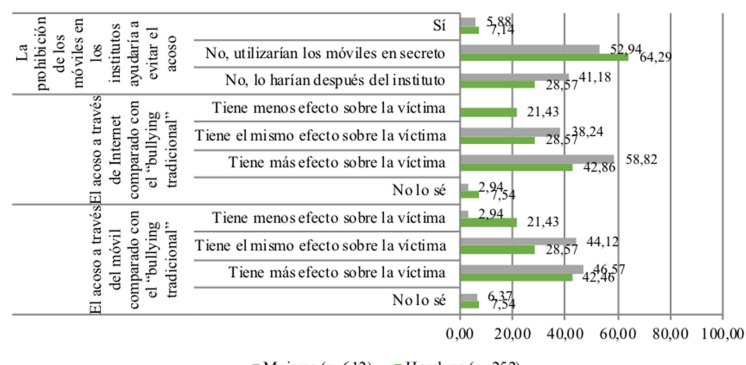

Figura 1. Percepción sobre los efectos del ciberbullying

\section{c) Frecuencia del acoso sufrido o ejercido a través del teléfono móvil e Internet}

En cuanto a la cantidad de veces que han sufrido o ejercido acoso a través del móvil (figura 2), la mayoría de los participantes (más del 69\%) indica que nunca lo han sufrido ni ejercido. Del porcentaje de estudiantes que indica que sí ha sufrido acoso a través del móvil, la mayoría de mujeres revela que este solo ha sucedido una o dos veces $(14,05 \%)$ mientras que para los hombres mayoritariamente esta situación se dilató en el tiempo, teniendo una frecuencia de una vez a la semana $(17,06 \%)$. Entre los estudiantes que indican que han ejercido acoso a través del móvil, la mayoría de mujeres revela que lo han hecho alrededor de una vez a la semana $(8,99 \%)$ mientras que los hombres indican que solo ha sucedido una o dos veces $(8,33 \%)$.

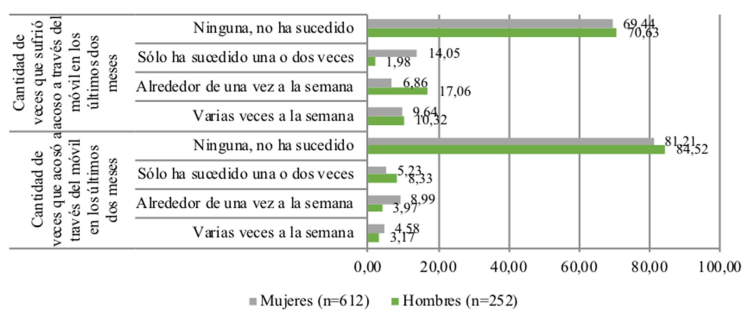

Figura 2. Frecuencia del acoso sufrido o ejercido a través del móvil

Referente a las veces que los participantes han sufrido o ejercido acaso a través de Internet (figura 3), la mayoría (más del 71\%) indica que nunca lo han sufrido ni ejercido. De los estudiantes que indican que sí que han sufrido acoso a través de Internet, la mayoría revela que este hecho se ha producido alrededor de una vez a la semana $(8,82 \%$ de mujeres y $14,29 \%$ de hombres). Los estudiantes que indican que han ejercido acoso a través de Internet, la mayoría de mujeres revela que lo han hecho varias veces a la semana $(5,88 \%)$ mientras que los hombres indican que ha sucedido alrededor de una vez a la semana $(2,78 \%)$.

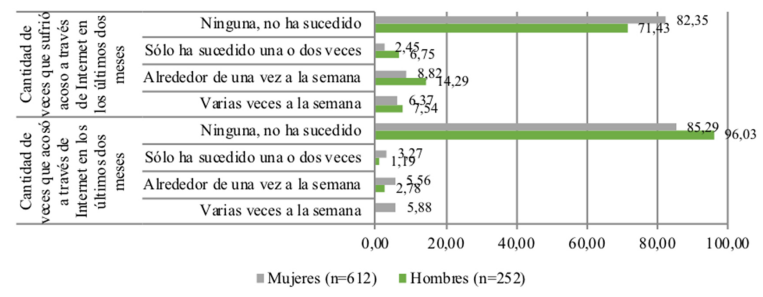

Figura 3. Frecuencia del acoso sufrido o ejercido a través de Internet

\section{d) Percepción ante el acoso}

De los participantes que indicaron que sufren acoso a través del móvil (187 mujeres y 74 hombres) (figura 4), la mayoría de hombres indican que cuando les acosan se sienten enfadados $(55,41 \%)$, solos $(50 \%)$ y les preocupa lo que los demás puedan pensar sobre lo que les está sucediendo (51,35\%). Cabe destacar que un porcentaje alto (51,35\%) indica no sentir nada cuando lo acosan. La persona que acosa a los hombres suele ser identificada por éstos como una chica de su curso $(37,84 \%)$, la duración del acoso sufrido suele ser de más de un año $(41,89 \%)$ y la forma en la que les acosan a través del móvil suele ser sobre todo por redes sociales (Instagram, twitter...) (50\%). En cuanto a la reacción que tienen ante el acoso, los participantes suelen ignorar lo que les sucede $(52,76 \%)$.

Por su parte, la mayoría de mujeres indican que cuando les acosan se sienten enfadadas $(57,75 \%)$, se sienten mal (38,50\%) y en menor medida les preocupa lo que los demás puedan pensar sobre lo que les está sucediendo (31,02\%). La persona que acosa a las mujeres suele ser identificada por éstas como principalmente un chico de su curso (41,18\%), la duración del acoso sufrido suele ser de dos o tres semanas $(55,61 \%)$ y la forma en la que les acosan es a través de SMS (41,18\%). En cuanto a la reacción que tienen ante el acoso, la mayoría de las participantes $(55,61 \%)$ ignoran lo que les pasa, aunque también un porcentaje alto $(42,92 \%)$ ha manifestado por el mismo medio a la persona que le acosa que dejase de hacerlo. 
Rodríguez Correa, Marisol; Rivadulla López, Juan Carlos. (2018). "Percepción y experiencias sobre el ciberbullying en estudiantes universitarios” en @tic. revista d'innovació educativa. Número 21 Otoño (Julio-Diciembre 2018), pp. 10-22.

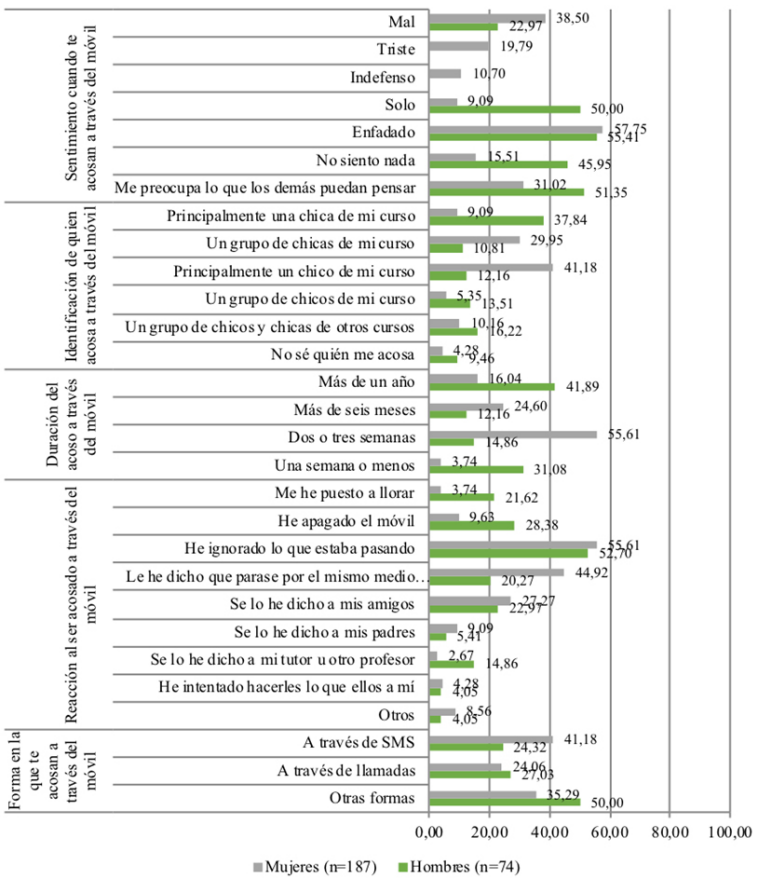

Figura 4. Percepción ante el acoso sufrido a través del móvil

De los participantes que indicaron que sufren acoso a través de Internet (108 mujeres y 72 hombres) (figura 5), a la mayoría de hombres les preocupa lo que los demás puedan pensar sobre lo que les está sucediendo (58,33\%). La persona que acosa por Internet a los hombres suele ser un grupo de chicos y chicas de otros cursos $(37,50 \%)$, la duración del acoso sufrido suele ser de una semana o menos (50\%) y la forma en la que les acosan a través de Internet suele ser por chats (59,72\%). En cuanto a la reacción que tienen ante el acoso, los participantes suelen ignorar lo que les sucede $(59,72 \%)$. Por su parte, la mayoría de mujeres indican que cuando les acosan se sienten solas (58,33\%) y les preocupa lo que los demás puedan pensar sobre lo que les está sucediendo (69,44\%). La persona que acosa a las mujeres suele ser identificada por éstas como principalmente un chico de su curso (53,70\%), la duración del acoso sufrido suele ser de más de seis meses $(36,11 \%)$ y la forma en la que les acosan es por chats principalmente $(66,67 \%)$. En cuanto a la reacción que tienen ante el acoso, la mayoría de las participantes $(47,22 \%)$ ignoran lo que les pasa.

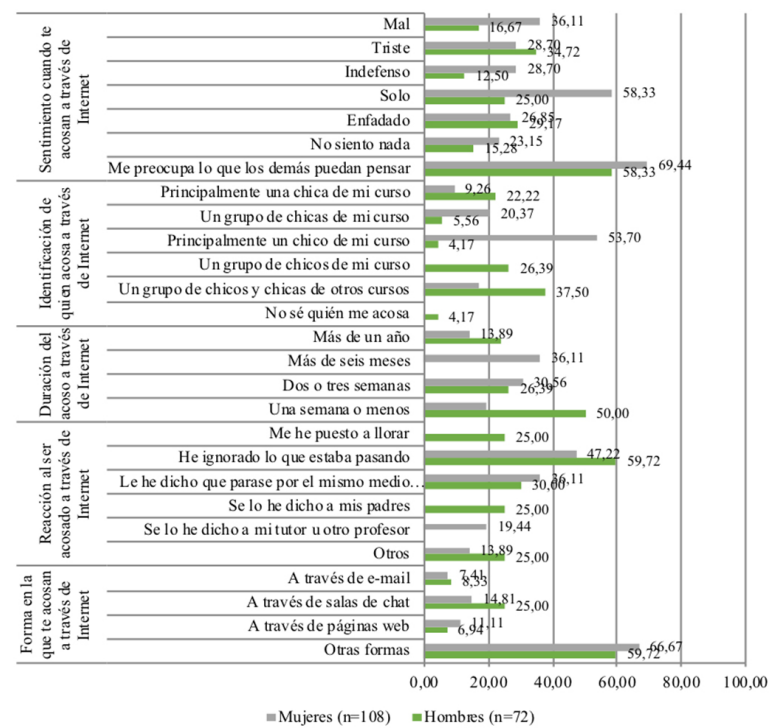

Figura 5. Percepción ante el acoso sufrido a través de Internet

A los participantes que indicaron ejercer acoso a través del móvil (115 mujeres y 39 hombres) e Internet (72 mujeres y 10 hombres) se les preguntó que cómo creían que se sentían las personas acosadas (figuras 6 y 7 ). La mayoría consideran que sus agredidos se sienten enfadados (más del $43 \%$ en el caso del móvil y más del $70 \%$ en el caso de Internet). Cabe destacar que, en la agresión a través del móvil, un pequeño grupo de participantes que se consideran agresoras señalan que sus víctimas no sienten nada $(8,70 \%$ de mujeres y $7,69 \%$ de hombres).

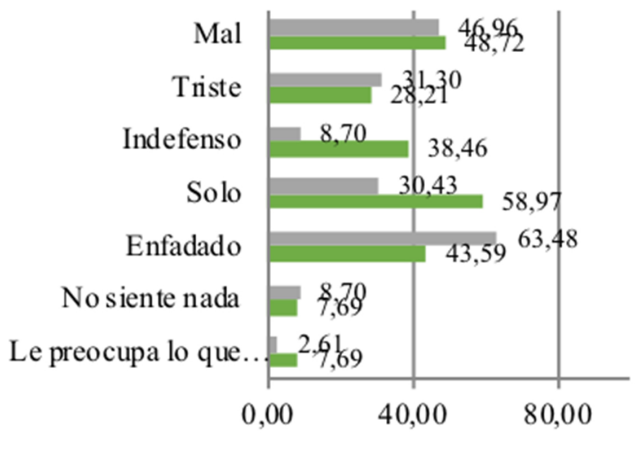

Mujeres $(\mathrm{n}=115) \quad \square$ Hombres $(\mathrm{n}=39)$

Figura 6. Percepción de cómo se siente la persona contra la que se ejerce acoso a través del móvil

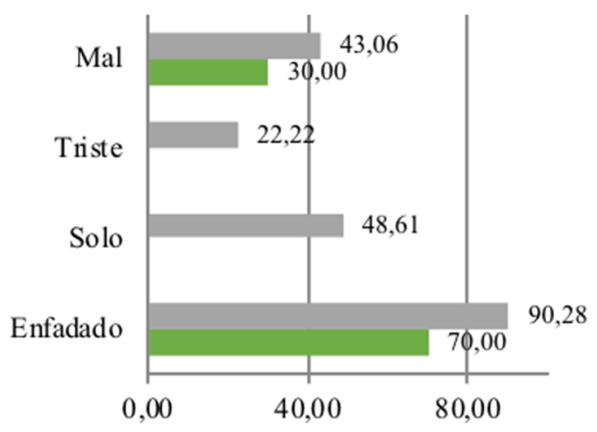

mujeres $(\mathrm{n}=72) \quad$ Hombres $(\mathrm{n}=10)$

Figura 7. Percepción de cómo se siente la persona contra la que se ejerce acoso a través de Internet 


\section{Discusión de los resultados}

Con nuestro trabajo de investigación hemos pretendido conocer la percepción y experiencia de un grupo de jóvenes universitarios sobre el ciberbullying. De los resultados obtenidos, podemos extraer una serie de reflexiones que nos permiten situarnos con mayor conocimiento ante el tema estudiado, el cual se ha convertido en un fenómeno muy problemático en nuestras instituciones educativas:

La mayoría del alumnado consultado en nuestro estudio indicó que el acoso a través del móvil y de Internet tiene más efecto sobre la víctima que el "bullying tradicional". Estos resultados coinciden con lo expuesto por Hinduja y Patchin (2009), cuando señalan que el acoso entre iguales llevado a cabo a través de las nuevas tecnologías produce importantes consecuencias en las víctimas, produciéndose una alteración en la convivencia escolar. De la misma forma, la mayoría del alumnado considera que prohibir los móviles en las instituciones educativas no ayudaría a evitar el acoso porque indican que se utilizarían estos dispositivos en secreto o fuera de la universidad. Cabe destacar, que un menor porcentaje de los estudiantes consultados consideran que el prohibir el uso de móviles en los centros educativos sería una buena opción para evitar el acoso.

La mayoría del alumnado consultado indicó que nunca han sufrido acoso ni han ejercido como acosadores; y solo un menor porcentaje de estudiantes expresó que sí ha sufrido acoso a través del móvil, pero con poca frecuencia. De la misma forma, solo un porcentaje pequeño de los alumnos indicó haber ejercido acoso a través del móvil alrededor de una vez a la semana en el caso de las mujeres, mientras que los hombres indicaron que solo ha sucedido una o dos veces.

En relación a Internet, la mayoría del alumnado señaló que nunca ha sufrido ni ejercido acoso a través de Internet; y solo un porcentaje muy pequeño de estudiantes señaló que sí lo ha sufrido acoso, pero con poca frecuencia. De la misma forma, los estudiantes que indicaron que habían ejercido acoso a través de Internet señalaron que lo habían hecho en varias ocasiones durante la semana en el caso de las chicas, mientras que los chicos apuntaban una periodicidad de una vez a la semana. Es importante destacar, que estos resultados coinciden con los expuestos anteriormente con relación al acoso a través del móvil.

La mayoría de los participantes de género masculino indicaron que, cuando han sufrido acoso a través del móvil no sentían nada cuando eran acosados. Según Tamres, Janicky \& Helgeson (2002) dado que el rol de género masculino se caracteriza por tener atributos más resolutivos y activos, es probable que los chicos adolescentes puedan utilizar con mayor frecuencia estrategias centradas en el problema y reevaluaciones positivas para tratar de cambiar experiencias negativas diarias que ellos creen que les provocan ciertos estados emocionales.

De la misma forma, un porcentaje alto de participantes de este mismo género indicó que, cuando les acosan, sienten enfado y que les preocupa lo que los demás puedan pensar con relación a lo que les está sucediendo. La mayoría de los hombres señalaron haber sido acosados por una chica de su curso, siendo la duración del acoso sufrido de más de un año y, producirse sobre todo en redes sociales (Instagram, Twitter...).

Por otra parte, la mayoría de las mujeres analizadas que indicaron que sí han sufrido acoso a través del móvil señalaron que, cuando les acosan se sienten enfadadas, se encuentran mal y, en menor medida, les preocupa lo que los demás puedan pensar sobre lo que les está sucediendo.

La mayoría de los participantes, tanto chicos como chicas, que indicaron ejercer acoso a través del móvil e Internet, consideran que sus agredidos se sienten enfadados. Estos resultados coinciden con lo expuesto por Ortega, Del Rey \& Casas (2012), cuando señalan que la cibervictimización se ha asociado en numerosos estudios con sentimientos negativos como la ira, el enfado, la tristeza, la desesperanza, el miedo, la culpa o la soledad.

\section{Conclusiones}

La implementación de medidas educativas para la prevención del ciberacoso en los centros educativos desde los primeros niveles es muy importante. Tal y como indicaron los alumnos consultados, y los resultados de otros estudios realizados sobre el tema, las víctimas del ciberacoso experimentan una serie de consecuencias tales como una falta de aceptación entre sus iguales, lo que conlleva en la mayoría de los casos a la baja autoestima, la depresión y el aislamiento social (Perren, Dooley, Shaw \& Cross, 2010; Sourander, BrunsteinKlomek, Ikonen, Lindroos, Luntamo, Koskelainen, Ristkari, \& Helenius, 2010; Zwierzynska, Wolke, \& Lereya, 2013; Nixon, 2014).

Los resultados del estudio aportan evidencia empírica con relación a algunas diferencias encontradas entre lo indicado por los hombres y mujeres participantes de nuestro estudio, como son las siguientes: las mujeres que indicaron ejercer alguna vez acoso a través del móvil lo hicieron alrededor de una vez a la semana, los hombres indicaron que solo ha sucedido una o dos veces. Así mismo, las mujeres señalaron que la persona que las acosa a través del móvil suele un chico de su curso al igual que los hombres consultados; sin embargo, los resultados difieren con respecto a estos en cuanto a la duración y medio a través del cual se produce el acoso; ya que en el caso de los hombres la duración del acoso sufrido es de más de un año produciéndose sobre todo en redes sociales como Instagram y Twitter, y en el caso de las mujeres la duración es de dos o tres semanas, siendo el medio a través del cual sufren acoso la mensajería SMS. Por otra parte, un porcentaje alto de chicos indicó que, cuando les acosan a través del móvil les preocupa lo que los demás puedan pensar con relación a lo que les está sucediendo, mientras que las chicas manifestaron que cuando les acosan se sienten enfadadas, se encuentran mal y, en menor medida, les preocupa lo que los demás puedan pensar sobre lo que les está sucediendo. Con relación al acoso a través de Internet, encontramos igualmente algunas diferencias en los resultados de nuestro estudio, mientras que la mayoría de los chicos que indicaron sufren acoso a través de Internet les preocupa lo que los demás puedan pensar sobre lo que les está sucediendo, un gran porcentaje de chicas indicó que, cuando les acosan, se sienten solas. De la misma forma, los chicos señalaron que la persona que les acosa por Internet suele ser un grupo de chicos y chicas de otros cursos, siendo la duración del acoso de una semana o menos, mientras que la persona que acosa a las chicas suele ser identificada por éstas generalmente como un chico de su curso, siendo la duración del acoso más de seis meses. Consideramos que es importante educar y sensibilizar a la sociedad en general acerca del tema del acoso y 
Rodríguez Correa, Marisol; Rivadulla López, Juan Carlos. (2018). "Percepción y experiencias sobre el ciberbullying en estudiantes universitarios” en @tic. revista d'innovació educativa. Número 21 Otoño (Julio-Diciembre 2018), pp. 10-22.

ciberacoso, siendo necesario continuar formando e informando de los beneficios de las TIC, así como de los riesgos que conlleva, y de las consecuencias sociales y legales que puede tener un mal uso de las mismas, "actuar sobre este problema y facilitar que el alumnado asuma los riesgos de este fenómeno, para que colabore en su erradicación, es responsabilidad de toda la comunidad educativa" (Giménez-Gualdo, Arnaiz-Sánchez, Cerezo-Ramírez, y Prodócimo, 2018).

\section{Bibliografía}

Álvarez-García, D., Núñez, J. C., Rodríguez, C., Álvarez, L., y Dobarro, A. (2011). Propiedades psicométricas del Cuestionario de Violencia Escolar Revisado (CUVE-R). Revista de Psicodidáctica, 16(1), 59-83.

Avilés, J. M. (2002). Bullying. Intimidación y maltrato entre alumnos. Bilbao: STEE-EILAS

Avilés, J.M. (2009). Ciberbullying. Diferencias entre el alumnado de Secundaria. Boletín de Psicología, 96, 79-96.

Beckman, L., Hagquist, C., \& Hellström, L. (2012). Does the association with psychosomatic health problems differ between cyberbullying and traditional bullying? Emotional and Behavioural Difficulties, $\quad 17, \quad 421-434$. https://doi.org/10.1080/13632752.2012.704228

Belsey, B. (2005). Cyberbullying: An emerging threat to the "always on" generation. Recuperado de http://www.cyberbullying.ca/pdf/feature_dec2005.p df.

Bisquerra, R. (coord.) (2004). Métodos de investigación educativa. Madrid: La Muralla.

Bringué, X., y Sádaba, Ch. (2010). Niños y adolescentes españoles ante las pantallas: rasgos configuradores de una generación interactiva. CEE Participación Educativa, 15, 86-104

Cabello, P. (2013). A Qualitative Approach to the Use of ICTs and its Risks among Socially Disadvantaged Early Adolescents and Adolescents in Madrid, Spain. Communications. The European Journal of Communication Research, 38, 61-83. http://doi.org/vnx

Calvete, E., Orue, L., Estévez, A., Villardón, L., y Padilla, P. (2010). Cyberbullying in adolescents: Modalities and aggressors' profile. Computers in Human Behavior, 26, 1128-1135. https://doi.org/10.1016/j.chb.2010.03.017

Calmaestra, J. (2011). Cyberbullying: prevalencia y características de un nuevo tipo de bullying indirecto (Tesis doctoral, Universidad de Córdoba). Servicio de Publicaciones de la Universidad de Córdoba.

Cerezo, F. (2009). Bullying: Análisis de la situación en las aulas españolas. International Journal of Psychology and Psychological Therapy, 9(3), 383394

Defensor del Pueblo-UNICEF (2007). Violencia escolar: el maltrato entre iguales en la Educación Secundaria Obligatoria 1999-2006. Madrid:
Servicio de Publicaciones del Defensor del Pueblo

Del Rey, R., Mora-Merchán, J., Casas, J., OrtegaRuiz, R., y Elipe, P. (2018). Programa "Asegúrate": Efectos en ciberagresión y sus factores de riesgo". Revista Comunicar, 56, XXVI. DOI: https://doi.org/10.3916/C56-2018-04

Durán-Segura, M., y Martínez-Pecino, R. (2015). Ciberacoso mediante teléfono móvil e Internet en las relaciones de noviazgo entre jóvenes. Revista Comunicar, 44, 159-167.

EUROSTAT (2017). Digital economy and society statistics - households and individuals. Recuperado de http://ec.europa.eu/eurostat/statisticsexplained/index.php/Digital_economy_and_society _statistics_households_and_individuals\#Internet_a ccess

Expósito, E., Navarro, E., Thoilliez, B., y López, E. (2010). Determinants of child well-being: A perspective from students of education. Comunicación presentada en la European Conference on Educational Research (ECER), Helsinki, Finlandia.

Finkelhor, D., Mitchell, K.J., \& Wolak, J. (2007). A Report on the Nation's Youth. Alexandria, Virginia: National Center for Missing \& Exploited Children.

Fundación Mutua Madrileña y Fundación Anar (2016). I Estudio sobre ciberbullying según los afectados. Recuperado de http://www.anar.org/wpcontent/uploads/2016/10/I-Estudio-sobreCiberbullying-seg\%C3\%BAn-losafectados.pdf

Garaigordobil, M. (2012). Bullying y Cyberbullying: Conceptualización, prevalencia y evaluación. Formación Continuada a Distancia (FOCAD). Edición Extraordinaria-Junio 2012. Consejo General Colegio Oficial de Psicólogos.

Garaigordobil, M., y Aliri, J. (2013). Ambivalent Sexism Inventory: Standardization and normative data in a sample of the Basque Country. Behavioral Psychology / Psicologia Conductual 21(1), 173186.

Garaigordobil, M. (2015). Ciberbullying en adolescentes y jóvenes del país vasco: Cambios con la edad. Anales de Psicología, 31(3), 10691076.

https://doi.org/10.6018/analesps.31.3.179151

Garaigordobil, M., Martínez, V., y Machimbarrena, J. (2017). Intervención en el bullying y ciberbullying: Evaluación del caso Martín. Revista de Psicología con Niños y Adolescentes, 4(1). Recuperado de http://www.revistapcna.com/sites/default/files/1518.pdf

Giménez-Guado, A., Arnaiz-Sánchez, P., CerezoRamírez, F., y Prodócimo, E. (2018) Percepción de docentes y estudiantes sobre el ciberacoso. Estrategias de intervención en Primaria y Secundaria. Revista Comunicar, 56, XXVI. https://doi.org/10.3916/C56-2018-03

Hinduja, S., y Patchin, J. (2009). Bullying beyond the Schoolyard: Preventing and Responding to 
Rodríguez Correa, Marisol; Rivadulla López, Juan Carlos. (2018). “Percepción y experiencias sobre el ciberbullying en estudiantes universitarios” en @tic. revista d'innovació educativa. Número 21 Otoño (Julio-Diciembre 2018), pp. 10-22.

Cyberbullying. Thousand Oaks, CA: Sage Publications

INTECO (2011). Estudio sobre hábitos seguros en el uso de smartphones por los niños y adolescentes españoles. Madrid: Observatorio de la Seguridad de la Información.

López, M. (2017). Acoso escolar y cibernético en estudiantes universitarios. Revista de Investigación en Educación, 15(1), 2017, pp. 1126. Recuperado de http://webs.uvigo.es/reined/

Martínez, R.A. (2007). La investigación en la práctica educativa: Guía metodológica de investigación para el diagnóstico y evaluación en los centros docentes. Madrid: Ministerio de Educación y Ciencia.

McMillan, J., y Schumager, S. (2005). Investigación educativa. Madrid: Pearson Addison Wesley.

Muñoz, J. (2016). Factores de riesgo en el acoso escolar y el ciberacoso: implicaciones educativas y respuesta penal en el ordenamiento jurídico español. Revista Criminalidad, 58(3), 71-86.

Nixon, C.L. (2014). Current perspectives: The impact of cyberbullying on adolescent health. Adolescent Health, Medicine and Therapeutics, 5, 143-158. https://doi.org/10.2147/AHMT.S36456

O'Brien, N., \& Moules, T. (2013). Not sticks and stones but tweets and texts: Findings from a national cyberbullying project. Pastoral Care in Education: International Journal of Psychology in Education, 31, 53-65. https://doi.org/10.1080/02643944.2012.747553

Oliveros, M., Amemiya, I., Condorimay, Y., Oliveros, R., Barrientos, A., y Rivas, B. (2012). Ciberbullying: Nueva tecnología electrónica al servicio del acoso escolar en alumnos de dos distritos de Lima, Perú. Anales de la Facultad de Medicina, 73(1), 13-8. https://doi.org/10.15381/anales.v73i1.804

Ortega, R., Calmaestra, J., y Mora-Merchán, J.A. (2007). Cuestionario cyberbullying. Recuperado de https://www.uco.es/laecovi/img/recursos/RFUY4M DDVCZWHkm.pdf

Ortega, R., y Mora-Merchán, J. (2008). Las redes de iguales y el fenómeno del acoso escolar: Explorando el esquema dominio-sumisión. Infancia y Aprendizaje, 31(4), 515-528. https://doi.org/10.1174/021037008786140922

Ortega-Ruiz, R., Del Rey, R., y Casas, J.A. (2012). Knowing, building and living together on internet and social networks: The ConRed cyberbullying prevention program. International Journal of Conflict and Violence, 6(2), 302-312. https://doi.org/10.4119/UNIBI/ijcv.250

Palacios, V., Polo, M., Felipe, E., León, B., y Fajardo, F. (2013). Tipología familiar y dinámica bullying/ciberbullying en educación secundaria. European Journal of Investigation in Health, Psichology and Education, 3(2), 161-170.

Palladino, B.E., Nocentini, A., \& Menesini, E. (2012). Online and offline peer led models against bullying and cyberbullying. Psicothema, 24(4), 634-639

Perren, S., Dooley, J., Shaw, T., \& Cross, D. (2010). Bullying in school and cyberspace: Associations with depressive symptoms in Swiss and Australian adolescents. Child and Adolescent Psychiatry and Mental Health, 4(28): 28-38. https://doi.org/10.1186/1753-2000-4-28.

Sabater Fernández, C., y López-Hernáez, L. (2015). Factores de Riesgo en el Ciberbullying. Frecuencia y Exposición de los Datos Personales en Internet. International Journal of Sociology of $\begin{array}{ll}\text { Education, } & 4(1),\end{array}$ http://dx.doi.org/10.4471/rise.2015.01

Sontag, L.M., Clemans, K.H., Graber, J.A., \& Lyndon, S.T. (2011). Traditional and cyber aggressors and victims: a comparison of psychosocial characteristics. Journal of Youth and Adolescence, 40, 392-404. https://doi.org/10.1007/s10964-0109575-9

Sourander, A., BrunsteinKlomek, A. B., Ikonen, M., Lindroos, J., Luntamo, T., Koskelainen, M., Ristkari, T., \& Helenius, H. (2010). Psychosocial risk factors associated with cyberbullying among adolescents. Archives of General Psychiatry, 67(7), 720-728. https://doi.org/10.1001/archgenpsychiatry.2010.79

Tangen, D., \& Campbell, M. A. (2010). Cyberbullying Prevention: One Primary School's Approach. Australian Journal of Guidance and Counselling, 20(2), https://doi.org/10.1375/ajgc.20.2.225.

Tamres, L.K., Janicki, D., \& Helgeson, V.S. (2002). Sex differences in coping behavior: a meta-analytic review and an examination of relative coping. Personality and Social Psychology Review, 6, 230. https://doi.org/10.1207/S15327957PSPR0601_1

Valverde, R. M., Fajardo, M. I. y Cubo, S. (2014). La violencia entre iguales en la adolescencia a través de las tecnologías de la comunicación e información. International Journal of Developmental and Educational Psychology, 1(5), 227-238.

Vandebosch, H., \& Van Cleemput, K. (2009). Cyberbullying among youngsters: profiles of bullies and victims. New Media \& Society 12, 11(8), 13491.371. https://doi.org/10.1177/1461444809341263

Yubero, S., Larrañaga, E., Navarro, R., y Elche, M. (2018) Padres, hijos e Internet. Socialización familiar de la red. Universitas Psychologica, 17, 2. http://dx.doi.org/10.11144/javeriana.upsy17-2.phis

Zwierzynska, K., Wolke, D., \& Lereya, T. S. (2013). Peer victimization in childhood and internalizing problems in adolescence: A prospective longitudinal study. Journal of Abnormal Child Psychology, 41(2), 309-323. https://doi.org/10.1007/s10802-012-9678-8. 
Rodríguez Correa, Marisol; Rivadulla López, Juan Carlos. (2018). "Percepción y experiencias sobre el ciberbullying en estudiantes universitarios” en @tic. revista d'innovació educativa. Número 21 Otoño (Julio-Diciembre 2018), pp. 10-22.

| Cita recomendada de este artículo

Rodríguez Correa, Marisol; Rivadulla López, Juan Carlos. (2018). “Percepción y experiencias sobre el ciberbullying en estudiantes universitarios". en @tic. revista d'innovació educativa. Número 21. Otoño (Julio-Diciembre 2018), pp. 1022.

\section{ANEXO}

El presente cuestionario forma parte de una investigación para conocer cómo se utilizan las nuevas tecnologías (Teléfono Móviles e Internet) en las relaciones entre los y las jóvenes como tú. Por favor responde de manera sincera a las preguntas, ya que el cuestionario es anónimo y nadie sabrá cuáles son tus respuestas.

Gracias por tu sinceridad y por dedicarnos tu tiempo.

\section{DATOS DE IDENTIFICACIÓN}

1. Sexo
a. Hombre
b. Mujer

2. Edad $\overline{\text { años }}$

3. Curso

4. Rama de conocimiento de los estudios que cursas:

5. Lugar de residencia:

6. ¿Tienes teléfono móvil? Sí __ No

7. ¿Tienes acceso a Internet en casa? Sí

8. ¿Tienes acceso a Internet desde tu móvil? sí

Ahora nos interesa saber si alguien se ha metido con alguien o lo ha acosado a través del Teléfono Móvil en la actualidad (como estudiante universitario). Ejemplo de meterse con otro o acosarlo a través de llamadas telefónicas: enviar o recibir llamadas desagradables, repugnantes o amenazantes; hacer y enviar fotos y/o videos por el móvil, fotos/imágenes repugnantes o vídeos, enviados a ti, o fotos/imágenes repugnantes o vídeos enviados a otros sobre ti; recibir o enviar mensajes de texto, SMS, abusivos por el móvil, etc. Primero te preguntamos si los has recibido y luego si tú mismo/a los han enviado.

Percepción sobre los efectos del ciberbullying

1. ¿Piensas que prohibir los móviles en los institutos ayudaría a evitar que se metan con alguien o lo acosen?
a. Sí
b. No, utilizarían los móviles en secreto.
c. No, lo harían después del instituto.

2. Piensas que el acoso a través del móvil, comparado con el "bullying tradicional" (meterse con alguien sin utilizar Internet ni el Móvil) ...
a. Tiene menos efecto sobre la víctima.
b. Tiene el mismo efecto sobre la víctima.
c. Tiene más efecto sobre la víctima.
d. No lo sé.

Frecuencia del acoso sufrido o ejercido a través del teléfono móvil e internet

3. ¿Cuántas veces se han metido contigo o te han acosado a través de tu móvil en los últimos dos meses?
a. Ninguna, no ha sucedido.
b. Sólo ha sucedido una o dos veces.
c. Alrededor de una vez a la semana.
d. Varias veces a la semana.

4. ¿Te has metido tú con alguien o has acosado, a través del móvil, a otras personas en los últimos dos meses?
a. Ninguna, no ha sucedido.
b. Sólo ha sucedido una o dos veces.
c. Alrededor de una vez a la semana.
d. Varias veces a la semana.

Percepción ante el acoso

5. ¿Cómo te sientes cuando otra persona se mete contigo o te acosa a través del móvil? (Para esta pregunta puedes marcar más de una opción).
a. No me ha pasado nada de eso.
b. Me siento mal. 
Rodríguez Correa, Marisol; Rivadulla López, Juan Carlos. (2018). “Percepción y experiencias sobre el ciberbullying en estudiantes universitarios” en @tic. revista d'innovació educativa. Número 21 Otoño (Julio-Diciembre 2018), pp. 10-22.

c. Me siento triste.

d. Indefenso, nadie puede ayudarme.

e. Solo, aislado.

f. Me siento enfadado

g. No me afecta, no siento nada

h. Me preocupa lo que los demás puedan pensar o hablar sobre mí.

6. ¿Quién se mete contigo o te acosa a través del móvil?
a. Nadie, no se meten conmigo
b. Principalmente una chica de mi curso.
c. Un grupo de chicas de mi curso.
d. Principalmente un chico de mi curso.
e. Un grupo de chicos de mi curso.
f. Un grupo de chicos y chicas de otros cursos.
g. No sé quién me acosa.

7. ¿Cuánto tiempo duró o dura el meterse contigo o el acosarte a través del móvil?

No se han metido conmigo usando el móvil
a. Duró más de un año
b. Duró más de seis meses
c. Duró dos o tres semanas
d. Una semana o menos

8. ¿Qué has hecho normalmente cuando alguien se ha metido contigo o te acosado a través del móvil? (Para esta pregunta puedes marcar más de una opción)
a. Nada, no me ha pasado nada de eso.
b. Me he puesto a llorar.
c. He apagado el móvil.
d. He ignorado lo que estaba pasando.
e. Le he dicho que parase por el mismo medio (llamada, SMS, etc.).
f. Se lo he dicho a mis amigos.
g. Se lo he dicho a mis padres.
h. Se lo he dicho a mi tutor $u$ otro profesor.
i. He intentado hacerles lo que ellos a mí.

9. ¿Cómo se meten contigo o te acosan a través del teléfono móvil? (Para esta pregunta puedes marcar más de una opción)
a. Nadie se mete conmigo de esa forma
b. A través de SMS (mensajes cortos)
c. Mensajes MMS (multimedia, vídeos, fotos)
d. A través de llamadas
e. De otro cómo): forma (escribe

10. ¿Cómo crees que se siente la persona con la que tú te metes o le acosas a través del móvil? (Para esta pregunta puedes marcar más de una opción).
a. No me meto ni acoso a nadie.
b. Se siente mal.
c. Se siente triste.
d. Se siente indefenso.
e. Se siete solo, aislado.
f. Se siente enfadado
g. No le afecta, no siente nada
h. Le preocupa lo que piensan los demás.

Ahora necesitamos conocer si alguien se ha metido contigo o te ha acosado, o tú te has metido o has acosado a alguien a través de Internet en la actualidad (como estudiante universitario). Son ejemplos de este tipo de cosas: e-mail en los que se meten contigo o te acosan; meterse con otro o acosar a través de salas de Chat; mensajes abusivos, desagradables o amenazantes a través de whatsapp u otros chat; páginas Web insultantes, en las que se revelen secretos o detalles personales que son agresivos, etc.

Percepción sobre los efectos del ciberbullying

11. Piensas que el acoso a través de Internet, comparado con el "bullying tradicional" (meterse con alguien sin utilizar Internet) ...
a. Tiene menos efecto sobre la víctima.
b. Tiene el mismo efecto sobre la víctima.
c. Tiene más efecto sobre la víctima.
d. No lo sé.

Frecuencia del acoso sufrido o ejercido a través del teléfono móvil e internet

12. ¿Cuántas veces se han metido contigo o te han acosado a través de Internet en los últimos dos meses?
a. Ninguna, no ha sucedido. 
Rodríguez Correa, Marisol; Rivadulla López, Juan Carlos. (2018). “Percepción y experiencias sobre el ciberbullying en estudiantes universitarios” en @tic. revista d'innovació educativa. Número 21 Otoño (Julio-Diciembre 2018), pp. 10-22.

b. Sólo ha sucedido una o dos veces.

c. Alrededor de una vez a la semana.

d. Varias veces a la semana.

13. ¿Te has metido tú con alguien o has acosado, a través de Internet, a otras personas en los últimos dos meses?
a. Ninguna, no ha sucedido.
b. Sólo ha sucedido una o dos veces.
c. Alrededor de una vez a la semana.
d. Varias veces a la semana.

Percepción ante el acoso

14. ¿Cómo te sientes cuando otra persona se mete contigo o te acosa a través de Internet? (Para esta pregunta puedes marcar más de una opción).

a. No me ha pasado nada de eso.

b. Me siento mal.

c. Me siento triste.

d. Indefenso, nadie puede ayudarme.

e. Solo, aislado.

f. Me siento enfadado.

g. No me afecta, no siento nada.

h. Me preocupa lo que los demás puedan pensar o hablar sobre mí.

15. ¿Quién se mete contigo o te acosa a través de Internet?
a. Nadie, no se meten conmigo.
b. Principalmente una chica de mi curso.
c. Un grupo de chicas de mi curso.
d. Principalmente un chico de mi curso.
e. Un grupo de chicos de mi curso.
f. Un grupo de chicos y chicas de otros cursos.
g. No sé quién me acosa.

16. ¿Cuánto tiempo duró o dura el meterse contigo o el acosarte a través de Internet?
a. No se han metido conmigo a través de Internet.
b. Duró más de un año.
c. Duró más de seis meses.
d. Duró dos o tres semanas.
e. Una semana o menos.

17. ¿Qué has hecho normalmente cuando alguien se ha metido contigo o te acosado a través de Internet? (Para esta pregunta puedes marcar más de una opción)
a. Nada, no me ha pasado nada de eso.
b. Me he puesto a llorar.
c. He ignorado lo que estaba pasando.
d. Le he dicho que parase por el mismo medio (E-mail, chat, etc.).
e. Se lo he dicho a mis amigos.
f. Se lo he dicho a mis padres.
g. Se lo he dicho a mi tutor $u$ otro profesor.
h. He intentado hacerles lo que ellos a mí.
i. He cerrado el programa/ventana y he seguido haciendo otra cosa con el ordenador.

18. ¿Cómo se meten contigo o te acosan a través de Internet? (Para esta pregunta puedes marcar más de una opción)
a. Nadie se mete conmigo de esa forma.
b. A través de E-mail.
c. A través de salas de chat.
d. A través de páginas web.
e. De
otra
forma
(escribe cómo):

19. ¿Cómo crees que se siente la persona con la que tú te metes o le acosas a través de Internet? (Para esta pregunta puedes marcar más de una opción).
a. No me meto ni acoso a nadie.
b. Se siente mal.
c. Se siente triste.
d. Se siente indefenso.
e. Se siete solo, aislado.
f. Se siente enfadado.
g. No le afecta, no siente nada.
h. Le preocupa lo que piensan los demás. MUCHAS GRACIAS POR TU PARTICIPACIÓN 